\title{
UNA NUEVA VISIÓN DE LAENSEÑANZA. El método integral en la enseñanza-aprendizaje del español como lengua extranjera en adultos
}

\author{
Mg. Araceli Cámero Room
}

\begin{abstract}
RESUMEN
Este artículo presenta el método integral como una prometedora alternativa en el proceso enseñanzaaprendizaje de un idioma extranjero para personas adultas; así como también, busca responder el cómo enfocar la explicación gramatical de la lengua extranjera en dicho proceso. Esto último, conlleva a poner atención en el alumno adulto, cuyas variables: edad, cultura social, preferencias $y$ experiencias son tomadas en cuenta con significante relevancia en el desarrollo del proceso enseñanza-aprendizaje de una lengua extranjera.
\end{abstract}

\section{ABSTRACT}

The present article presents the integral method as a very interesting alternative in the teaching and learning process of a foreign language far adu lt s. lt has also the purpose to focus on the grammatical explanation of the fareign language in such process, which leads to center on the adult student, which variables such as: age, social culture, preferences and experiences are taken into account with a vital relevance on the development of the learning -teaching process of aforeign language.

\section{PALABRAS CLAVE}

Lengua extranjera, factores lingüísticos y extralingüísticos, enfoque gramatical, adquisición, aprendizaje en adulto.

\section{KEYWORDS}

Foreign language, linguistic and extra linguistic factors, grammatical approach, acquisition, learning far adu lt s.

\section{Introducción}

I mportante s han sido los aportes de las diferentes teorías sobre la adquisición y/o aprendizaje de la lengua materna; sin embargo, los métodos y/o enfoques, basados en dichas teorías, hasta el momento no han cubierto las expectativas para llevar a cabo con éxito el desarrollo del proceso enseñanza aprendizaje de una lengua extranjera en personas adultas.

Los métodos que se encuentran enel mercado, hoy en día, mantienen un enfoque comunicativo natural y espontáneo; el cual, bajo la creencia de que el aprendizaje de reglas gramaticales inhibe el desarrollo de las habilidades lingüísticas en el alumno, prohíben cualquier referencia o explicación gramatical enla lengua materna y sugiere que esta sea procesada por el mismo alumno. Por ello dejan de lado la enseñanza formal de la lengua (la gramática) lo cual deja al estudiante adulto sin la explicación requerida y provoca en él confusiones y frustraciones.

\section{Influencia de la lingüística en la enseñanza delenguas extranjeras}

Es preciso subrayar la determinante influencia que la lingüística ha tenido en el campo de la lingüística aplicada, y de la linguística aplicada en la enseñanza de lenguas extranjeras. Todas las investigaciones 
acerca de la lengua han contribuido en la enseñanza de lenguas extranjeras y en la elaboración de materiales, así como también en la orientación pedagógica

José Manuel Vez afirma que el conoC1m1ento lingüístico ha contribuido con mucha fuerza en la evolución y naturaleza de los conceptos lingüísticos. Menciona la importancia del significado frente a la estructura, la semántica frente a la sinta xis, y el contexto socio sítuacíonal frente a los patrones de repetición mecánica.

Hoy en día, el método que se encuentra en las escuelas, universidades e instituciones culturales, mantiene un enfoque comunicativo. Este está basado en una teoría lingüística, la cual considera a la lengua como un sistema utilizado por los seres humanos con elobjetivo decomunicarse entre sí.

Asimismo, con el auge de este enfoque hubo quienes radicalizaron la idea de que lo esencial en el proceso enseñanz a-aprendi zaje eran las sí tu aciones comunicativas reales, naturales y espontáneas, considerando de esta manera que el aprendizaje de una lengua extranjera debería impartirse de la misma forma como se adquiere la lengua materna. Esto llevó a los especialistas a dejar de lado la enseñanza del aspecto formal dela lengua.

\section{Importancia de la gramática en el proceso enseñanza-aprendizaje de una lengua extranjera en personas adultas y cómo debe ser enfocada}

En términos generales, la gramática se define como el estudio científico del lenguaje. Hace mención a la competencia lingüística, a la estructura y al conocimiento de los contenidos fonológicos, morfológico, sintáctico y semántico que todo hablante debeposeer.

El término gramática, con relación a la enseñanza de lenguas extranjeras, ha sido uno de los temas más debatidos mundialmente, la importancia que se le ha concedido a este tema, siempre se ha visto influenciado por los diferentes métodos de enseñanza.

De acuerdo a una formación humanística clásica, el método de la gramática y traducción se basó, como su nombre así lo índica, en la enseñanza de la gramática y en la práctica de la traducción, teniendo acceso de este modo a la lectura de una lengua extranjera . Este método como se sabe, parte con la definición de
Elmétodo integralenlaenseiianza aprcndizajcdelespañolcomo lenguat:xtranjeraenadultos

clases de palabras y sigue con las reglas de su funcionamiento a través del procedimiento deductivo. A este método le siguió el método directo, el cual se caracterizaba en aceptar que el proceso de adquisición de una lengua extranjera era similar a la lengua materna.

El término directo hace mención al empleo de la lengua extranjera en todo el proceso de enseñanza y prohíbe usar, como intermediaria, la lengua materna en clase. Es en esta época donde se mantiene el concepto de lengua como estructura y el concepto conductista . la enseñanza -aprendi zaje de la gramática se desarrollaba mediante repetición de estructuras, método que, de acuerdo a numerosas investigaciones, quedó a un lado para pasar a la enseñ anz a-aprendi zaje de una lengua con significado (Vez,1980).

Es a partir de los años setenta y ochenta, con la aparición de las corrientes lingüísticas apoyadas en la etnolingüística, psicolingüística, socíolingüístíca, la pragmática y la lingüística de texto; y con la aparición, en Europa, del enfoque comunicativo revoluciona todo el esquema de la metodología de lenguas extranjeras, con la esperanza de encontrar la forma "correcta" de enseñarlas, que vuelve a plantear la pregunta, si es determinante la enseñanza de gramática en los cursos de lengua extranjera y cómo debe ser enfocada (Finocc hiaro,1983;Stern,1983).

la enseñanza de la gramática tiene defensores y detractores. Algunos estudiosos consideran que la gramática es determinante en la enseñanzaaprendizaje de una lengua extranjera, otros piensan que la instrucción gramatical se debe erradicar totalmente (Canale, 1983; Belichon, 1994 y Camps, 1998).

Diversas investigaciones afirman que el uso y comprensión de una lengua dependerá del nivel de competencia que posea el alumno; por esto, es importante desarrollar la competencia comunicativa, como también la reflexión de la lengua (competencia lingüística) en el desarrollo del proceso enseñanza-aprendizaje de una lengua ext ranjera.

Con el auge del enfoque comunicativo se fue dejando de lado la enseñanza de la gramática, pues se radicalizó la idea de que lo esencial en el proceso enseñanza-aprendizaje de una lengua extranjera era considerar las situaciones comunicativas naturales, reales y espontáneas. Sin embargo, se sabe que en el proceso aprendizaje de una lengua extrajera, la 
acerca de la lengua han contribuido en la enseñanza de lenguas extranjeras y en la elaboración de materiales, así como también en la orientación pedagógica.

José Manuel Vez afirma que el conoc1m1ento lingüístico ha contribuido con mucha fuerza en la evolución y naturaleza de los conceptos lingüísticos . Menciona la importancia del significado frente a la estructura, la semántica frente a la sintaxis, y el contexto socio situacional frente a los patrones de repetición mecánica.

Hoy en día, el método que se encuentra en las escuelas, universidades e instituciones culturales, mantiene un enfoque comunicativo. Este está basado en una teoría lingüística, la cual considera a la lengua como un sistema utilizado por los seres humanos con elobjetivo de comunicarse entre sí.

Asimismo, con el auge de este enfoque hubo quienes radicalizaron la idea de que lo esencial en el proceso enseñanz a-aprendi zaje eran las situaciones comunicativas reales, naturales y espontáneas, considerando de esta manera que el aprendizaje de una lengua extranjera debería impartirse de la misma forma como se adquiere la lengua materna. Estollevó a los especialistas a dejar de lado la enseñanza del aspecto formal dela lengua.

\section{Importancia de la gramática en el proceso enseñanza-aprendizaje de una lengua extranjera en personas adultas y cómo debe ser enfocada}

En términos generales, la gramática se define como el estudio científico del lenguaje. Hace mención a la competencia lingüística, a la estructura y al conocimiento de los contenidos fonológicos, morfológico, sintáctico y semántico que todo hablante debeposeer.

El término gramática, con relación a la enseñanza de lenguas extranjeras, ha sido uno de los temas más debatidos mundialmente, la importancia que se le ha concedido a este tema, siempre se ha visto influenciado por los diferentes métodos de enseñanza.

De acuerdo a una formación humanística clásica, el método de la gramática y traducción se basó, como su nombre así lo indica, en la enseñanza de la gramática y en la práctica de la traducción, teniendo acceso de este modo a la lectura de una lengua extran jera. Este método como se sabe, parte con la definición de
El método integral en la enseiianza aprcndizajc del es pañol co mo lengua extranjera en adulros

clases de palabras y sigue con las reglas de su funcionamiento a través del procedimiento deductivo. A este método lesiguió el métododirecto, el cual se caracterizaba en aceptar que el proceso de adquisición de una lengua extranjera era similar a la lengua materna.

El término directo hace mención al empleo de la lengua extranjera en todo el proceso de enseñanza y prohíbe usar, como intermediaria, la lengua materna en clase. Es en esta época donde se mantiene el concepto de lengua como estructura y el concepto conductista. La enseñanza-aprendizaje de la gramática se desarrollaba mediante repetición de estructuras, método que, de acuerdo a numerosas investigaciones, quedó a un lado para pasar a la enseñanz a-aprendi zaje de una lengua con significado (Vez,1980).

Es a partir de los años setenta y ochenta, con la aparición de las corrientes lingüísticas apoyadas en la etnolingüística, psicolingüística, sociolingüística, la pragmática y la lingüística de texto; y con la aparición, en Europa, del enfoque comunicativo revoluciona todo el esquema de la metodología de lenguas extranjeras, con la esperanza de encontrar la forma "correcta" de enseñarlas, que vuelve a plantear la pregunta, si es determinante la enseñanza de gramática en los cursos de lengua extranjera y cómo debe ser enfocada (Finocchiaro, 1983;Stern,1983).

La enseñanza de la gramática tiene defensores y detractores. Algunos estudiosos consideran que la gramática es determinante en la enseñanza aprendizaje de una lengua extranjera, otros piensan que la instrucción gramatical se debe erradicar totalmente (Canale, 1983; Belichon, 1994 y Camps, 1998).

Diversas investigaciones afirman que el uso y comprensión de una lengua dependerá del nivel de competencia que posea el alumno; por esto, es importante desarrollar la competencia comunicativa, como también la reflexión de la lengua (competencia lingüística) en el desarrollo del proceso enseñanza-aprendizaje de una lengua extranjera.

Con el auge del enfoque comunicativo se fue dejando de lado la enseñanza de la gramática, pues se radicalizó la idea de que lo esencial en el proceso enseñanza-aprendizaje de una lengua extranjera era considerar las situaciones comunicativas naturales, reales y espontáneas. Sin embargo, se sabe que en el proceso aprendizaje de una lengua extrajera, la

\section{1 un1Fé}


enseñanza de la gramática ayuda a satisfacer las necesidades metalingüísticas de los discentes adultos; puesto que el alumno adulto ya ha alcanzado un desarrollo cognitivo y tiende a realizar comparaciones conlalengua materna (Ortega,1990).

El conocuruento lingüístico se obtiene bien por adquisición o bien por apren dizaje. Krashen (1977) menciona que los niños adquieren el conocimiento lingüístico mediante modelos que ponen en funcionamiento la adquisición de una lengua; donde, de acuerdo a Bley Vroman (1980), intervienen mecanismos del cerebro que forman parte del área específica del lenguaje; lo que no ocurre con el adulto y por tal razón, en este caso, se habla de aprendizaje de una lengua, donde la enseñanza de la gramática se realizará de manera contextualizada considerando no solo reglas de construcción sino también de uso y teniendo en cuenta como propósito fundamental el perfeccionamientodela competencia comunicativa.

Por lo tanto, a la gramática se le debe dar un lugar en elproceso enseñanza-aprendizaje, considerando que en la enseñanza de lenguas extranjeras, la gramática es un medio y no un fin.

Corder (1973) muestra un esquema sobre las diferentes intenciones con respecto al enfoque gramatical:

Esquema relacional de autor, lector y propósito de la gramática

\begin{tabular}{|c|c|c|}
\hline Autor & Lector & Propósito de la gramática \\
\hline Lingüista & Lingüista & Ilustrar y validar una teoría sintáctica particular. \\
\hline Lingüista & $\begin{array}{l}\text { Estudiante } \\
\text { Lingüística }\end{array}$ & $\begin{array}{l}\text { Enseñar teoría sintáctica inductivamente a través } \\
\text { de su aplicación a un idioma. }\end{array}$ \\
\hline $\begin{array}{l}\text { Lingüista } \\
\text { Aplicado }\end{array}$ & $\begin{array}{l}\text { Hablante } \\
\text { Nativo } \\
\text { Culto }\end{array}$ & $\begin{array}{l}\text { Sistematizar y "nominazliar" los conocimientos } \\
\text { implicitos del lector. }\end{array}$ \\
\hline $\begin{array}{l}\text { Lingüista } \\
\text { Aplicado }\end{array}$ & $\begin{array}{l}\text { Docente de la lengua } \\
\text { materna }\end{array}$ & $\begin{array}{l}\text { Sistematizar el conocimiento implícito o } \\
\text { explicito del lector en una forma } \\
\text { pedagógicamente apropiada. }\end{array}$ \\
\hline $\begin{array}{l}\text { Lingüista } \\
\text { Aplicado }\end{array}$ & $\begin{array}{l}\text { Docente de una } \\
\text { segunda lengua }\end{array}$ & $\begin{array}{l}\text { Sistematizar el conocimiento de una manera que } \\
\text { sea pertinente para hablantes no nativos. }\end{array}$ \\
\hline
\end{tabular}

El cuadro anterior deja en claro que en la enseñanza, aprendizaje de una lengua extranjera se debe considerar a los alumnos como aprendices de una lengua y no como futuros lingüistas y ya Sweet (1899), añadía que es necesario distinguir entre los términos de "gramática práctica" y "gramática de referencia".

Es fácil observar que la apanc1on de distintos métodos en el mercado educativo se ha producido siguiendo dos extremos; por un lado, el método
El método integral en la enseñanza -aprend iz aje del español como lengua extranjera en adultos

gramática tradicional, y por el otro extremo, el método directo o natural. A partir de ahí el resto de los métodos aparecidos hasta hoy en el mercado educativo han estado moviéndose entre estas dos concepciones opuestas sobre el aprendizaje y enseñanza delenguas.

Sin embargo, se puede declarar que cada uno de ellos ofrece tan solo soluciones parciales a los problemas que se desarrollan en el proceso enseñanzaaprendizaje en un salón de clases. Esto se debe a que cada método se ha ido elaborando de forma singular o aislada, es decir, sin tomar en cuenta las bondades de los métodos que iban quedando atrás (Sánchez, 1997).

La experiencia ilustra a los profesionales de la didáctica que mientras se tenga una visión más amplia y plural dela realidad en la formulación de un método este tendrá mejores resultados y más oportunidades para permanecer en el mercado educativo por más tiempo . El análisis y la experiencia de la historia de los métodos en la enseñanza de idiomas extranjeros, orienta a no cometer el error de partir del vacío y considerar en todo momento los elementos de los métodos anteriores.

De ahí que el método integral no parte del vacío y su punto de partida es el método comunicativo del cual podría decirse que viene a ser la base en la cual descansa. Se ha tomado como base el método comunicativo, pues este contiene elementos que difícilmente podrían ser eliminados ele la docencia de lengua. El primer elemento es el lenguaje, el cual se caracteriza por su función comunicativa. El segundo es el mensaje transmitido a otra persona, ele ahí la importancia del contenido y el significado; y el tercer elemento, vienen a ser los protagonistas del proceso enseñanza-aprendi zaje, los cuales son:el docente y el discente.

En el método comunicativo, aún existen algunos elementos que cuando han sido considerados teóricamente en el proceso enseñanza-aprendi zaje, no se cumplen a cabalidad en la práctica y esta omisión trae consigo problemas que se presentan hasta hoy al utilizar dicho método. Estos elementos son:enelaspecto cognitivo, elproceso deenseñanz,a aprendizaje que está enfocado hacia una visión significativa; sin embargo el contenido se encuentra descontextualizado o se usan materiales que no son propios de acuerdo a la edad de los alumnos. En cuanto al aspecto psicológico, la relación entre docente y cliscente y el ambiente generado en el salón 
de clase muchas veces no es el apropiado, puesto que se presentan situaciones problemáticas que el docente no sabe cómo resolver; por lo que el docente con una capacitación intelectual y psicológica vendría a ser determinante para llevar a cabo el proceso con óptimos resultados. La carencia de idea de integración del método comunicativo, es lo que pone de manifiesto las limitaciones de este y otros métodos en el mercado educativo.

En los últimos años, se ha acentuado el carácter integrador de los valores humanos, esto se debe a muchos factores. Uno de ellos es la aceptación de la variedad no como algo que representa o amenaza, sino como algo que contribuye a enriquecer al hombre y a su entorno. La democracia exige que los unos respeten a los otros, estableciendo así un hábito de respeto mutuo entre todos los miembros de una sociedad.

En el caso del proceso enseñanza-aprendizaje el respeto se concreta de manera especial en el alumno. Este no era considerado en la tradición educativa; sin embargo, en una visión integral de la enseñanza, no considerar al alumno carece de justificación. El alumno es un agente no solamente indispensable, sino el principal, ya que la única razón de ser de la docencia es él(Goodman, 1999).

De acuerdo a la teoría psicológica en la cual se basa el método integral: el ser humano que aprende es, antes que nada, un ser humano, fruto de la conjunción de aspectos cognitivos, afectivos y físicos. $\mathrm{El}$ aprendizaje es un proceso activo que requiere la colaboración activa del individuo. Para que ésta sea más eficaz se precisa la participación responsable y autónoma de la persona con todas sus potencialidades. En el aprendizaje inciden la dimensión cognitiva, la afectiva y el entorno, tanto consciente como inconscientemente .

Por otro lado, la teoría pedagógica añade que el aprendizaje resulta de un proceso, en el que la mente desempeña un papel clave, pero no único; ya que este se adquiere con la ayuda de procesos mentales, mediante la consolidación a través de la repetición de actos del habla o de prácticas escritas. La práctica, en sus distintas modalidades, es indispensable para retener lo aprendido.

En cuanto a los principios de este método en la enseñanza, ésta debe ser participativa y democrática . El profesor es el responsable de la organización y actúa como ayuda y promotor de la enseñanza. Las actividades deben ser variadas y el error debe considerarse como normal a lo largo del aprendizaje; debe tolerarse hasta cierto punto, aunque debe evitarse su consolidación, especialmente si incide gravemente en la comprensión del mensaje (Sánchez).

El enfoque integral hace referencia que en el proceso aprendizaje de una lengua es necesario aprender de la lengua y a través de la misma, haciendo uso funcional del lenguaje, con ayuda de contextos auténticos y guiado por propósitos personales del aprendiz para satisfacer sus propias necesidades (Goodman,1989).

Este enfoque descansa sobre la base de la psicología cognitiva, la cual se centra en los procesos internos que realiza el individuo, consiste en adquirir un control consciente de las estrategias del lenguaje y elementos fonéticos, lexicales y gramaticales. Su principal exponente es el constructivismo, el cual se fundamenta en que el conocimiento existe en la mente.

Por todoesto, cabeseñalary afirmar quelaenseñanza integral del lenguaje, introducido por el investigador, profesor universitario, Kenneth Goodman, se basa firmemente en centrar el enfoque con un pensamiento humanístico-científico, donde la persona ocupa la primera atención; puesto que el proceso de enseñanza-aprendizaje integral está basado en una teoría lingüística, psicológica, pedagógica, y sociolingüística.

Asimismo, el enfoque integral centra su atención en la interdependencia que existe en el desarrollo cognoscitivo y lingüístico, así como también se enfoca hacia una enseñanza real, con significado y funcional, motivando, de esta manera, al estudiante a tomar decisiones y asumir riesgos (Goodman).

\section{El Método Integral en el proceso enseñanza-aprendizaje del Español como lengua extranjera en alumnos adultos}

El carácter integrador de las actividades es fundamental en el proceso enseñanza-aprendizaje de una lengua extranjera en alumnos adultos; por lo tanto, lo comunicativo como lo lingüístico presentan el mismo nivel de importancia en lo que respecta al método integral.

La sistematización, coherencia de elementos y actividades de estemétodo logran ese todo complejo que es la competencia comunicativa; puesto que se nutre e integra, no reúne ni toma elementos de 
métodos anteriores más bien los integra con la finalidad de lograr una competencia comunicativa de la lengua estudiada. Competencia que se encuentra integrada por varios componentes que al prescindir de ellos propiciaría necesariamente la pérdida de elementos comunicativos. Se puede concluir que un método integral es un método comunicativo, puesto que apunta hacia el logro de la competencia comunicativa . El término integral aduce que en el logro de tal finalidad deben ponerse en acción todas las técnicas que el análisis de la comunicación, mediante el lenguaje, revela como adecuados (Sánchez, 1997).

Así pues, centrados en una clase con estudiantes adultos, el método integral no rechaza una explicación gramatical sólo por el hecho de ser propia de un método tradicional, ni un ejercicio de repetición por haber sido característico de la metodología audio-oral. Todo lo contrario, tendrá en cuenta las actividades cognitivas las cuales tienen como finalidad elevar al nivel consciente los temas que son objeto de enseñanza o aprendizaje.

Por otro lado, es verdad que los problemas gramaticales han recibido demasiado énfasis en alguna metodología de clase tradicional; sin embargo, esto no debe ser causa de rechazo. Actualmente, en el mercado educativo existen libros que ofrecen numerosas e interesantes sugerencias sobre cómo enseñar la gramática de manera más amena y variada, lo cual contribuirá al aprovechamiento de las clases y el alumno sentirá que cada clase concluida habrá sido aprovechada al max1mo. Haciendo referencia a esto, el alumno adulto quiere conocer de modo absolutamente consciente la forma correcta de cada mensaje, no se conforma con que este llegue "a medias" y quiere racionalmente entender dónde se equivocó para así no repetir el mismo error. Por lo tanto, necesita volver a la fuente que pudo haber originado el error, sea interferencia de su propioidioma o por confusión dereglas gramaLicales delidioma extranjero.

Con respecto a la sensibilidad que se presenta en una clase de enseñanza-aprendizaje de una lengua extranjera en adultos, la experiencia contribuye a afirmar que la mayoría de los adultos tiene la percepción de estar cometiendo errores. Ellos mismos "sienten" que lo que están expresando no es correcto, no es preciso, no se ajusta a las reglas gramaticales que intentan aplicar, o que aquello que
El método integral en la enseñan za aprendizaj c del espart: I como le ngua extranjera en adul tos

quieren expresar no coincide con el mensaje emitido. Esta percepción les provoca incomodidad y dicho por ellos mismos, una sensación de incertidumbre y de no estar expresándose como adultos. De ahí, que al ser el enfoque integral, humanístico y científico, enfatiza la necesidad de comprender esta inquietud y solucionarla, entendiendo que aquel malestar no permitirá que el estudiante aproveche su capacidad de aprendizaje y, por ende, sentirá frustración.

El estudiante adulto, a través de su meta cognición, reconoce cuando no sabe. En el caso del aprendizaje de un idioma extranjero, su aprendizaje debe ser integral, realyrelevante, asícomotambiénfuncional puessedeberátenerencuenta elcontexto desuuso. Para esto, se requiere de un instructor experimentadoy conocedor del tema dela enseñanza en lenguas extranjeras en estudiantes adultos, un instructor que sepa cómo observar al estudiante adulto, cómo ver y guiar sus potencialidades y necesidades; así también, precisar el momento en el que los alumnos se encuentran preparados para el aprendizaje y hasta dónde podrán llegar con poca ayuda. A esto Vigotsky, psicólogo ruso, denominala zona de desarrollo próximo. El profesor experimentado sabe cómo detectar esa zona y cómo ayudaralalumnoparapoderguiarlo eficientementey poder enseñarle a aprender y no frustrarlo con exigencias o técnicas no adecuadas a las características del estudiante en cuestión.

Mencionadas las diferencias entre la manera en que se desenvuelve el aprendizaje de una lengua materna y/o lengua extranjera en un niño y en un adulto, no es acertado pretender aplicar la misma metodología de enseñanza a ambos .

Una de las actividades comunicativas más difícil de alcanzar en el proceso enseñanza-aprendizaje de una lengua extranjera en alumnos adultos es la expresión oral, ya que muchos estudiantes deben poner en práctica todo lo lingüísticamente y extralingüísticamente aprendido . Además, se debe tener en cuenta los factores personales como la timidez o el sentir de hacer el ridículo.

El siguiente cuadro demuestra cómo respondieron un examen de presentación oral los alumnos adultos de un nivel básico de español, sometidos al método integral y los alumnos adultos sometidos al método comunicativo. 


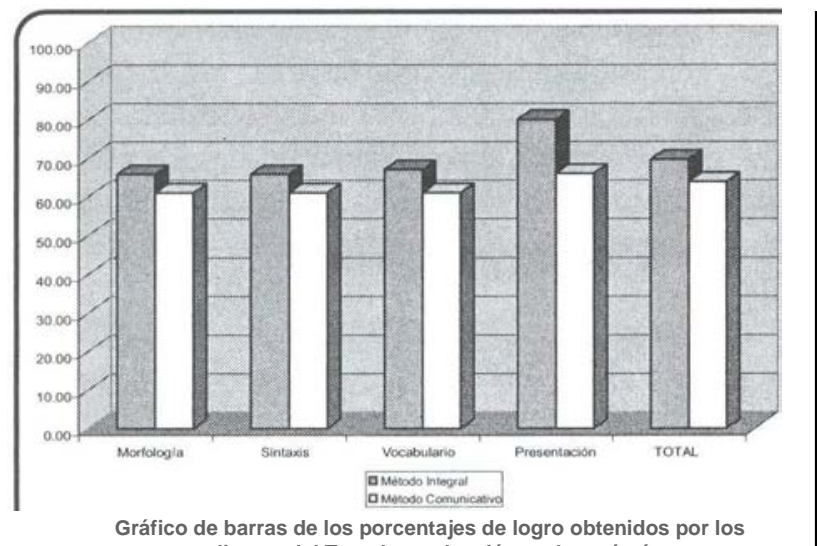

Gráfico de barras de los porcentajes de logro obtenidos por los estudiantes del Test de producción oral, según áreas

Estos resultados son producto de un trabajo sistematizado y coherente de elementos y actividades; no existieron prohibiciones ni restricciones deningunaíndoley se toleróunnivelde errores, el criterio moderador en todo el proceso fue el logro de la eficacia comunicativa en cada alumno. El carácter integrador de las actividades fue fundamental, puesto que elalumnoentodomomento del desarrollo del proceso enseñanza aprendizaje, contó con el apoyo de elementos lingüísticos y extralingüísticos; $y$, de esta manera, se encontró cognitiva y emocionalmente preparado para rendir una presentación oral. "El aprendizaje tiene que ver con la persona en la tonalidad de las diversas facetas que la integran... dimensión intelectual y cognitiva como la dimensión afectiva" (Sánchez, 2000).

\section{Conclusiones}

Es necesario tomar en cuenta, al diseñar programas de aprendizaje destinados para adultos, las necesidades, expectativas y las competencias lingüísticas y socioculturales que estos traen consigo; pues la integración de todos estos elementos determinará el buen desarrollo y la obtención de óptimos resultados en el proceso aprendizaje de una lengua extranjera.

En cuanto a los factores lingüísticos y extralingüísticos en el proceso enseñ anz aaprendizaje de una lengua extranjera, ambos deberán ser tomados en cuenta con significativa relevancia. En el factor lingüístico se ha considerado que la enseñanza de la gramática es determinante para el aprendizaje de idiomas en edad adulta. Y en el factor extralingüístico, lo psíquico, físico, y emocional (autoestima, personalidad, edad, origen del estudiante) deberá ser considerado paralelamente relevante en el proceso de enseñanza-aprendizaje de un idioma extranjero.

\section{REFERENCIAS}

Bley Vroman, R. (1980). The logical problem of foreign language learning. Oxford: University Press.

Brunner, Jerome (1966). Teorías cognitivas de aprendizaje. Massac husett s: Harvard University Press.

Cerny, Jiry (2006). Historia de la lingiiística. Madrid: Universidad de Extremadura.

Corder, S.P.(1973).Introducción a la lingüística aplicada. Madrid: Penguin.

Chomsky, Noam (1988). El lenguaje y los problemas del conocimiento. Madrid: Visor.

De Greve, Marcel; Van Passel, Frans (1971). Lingüística y enseñanza de lenguas extranjeras. Madrid: Fragua.

Ellis, R. (1996). The study of second language acquisition. Oxford: University Press.

Goldberg, F. (2008). Enseñanza en adultos. Barcelona: Paidós.

Goodman, Kenneth (1996). El lenguaje integral: Un camino fácil para el desarrollo del lenguaje. li ma: Centro Peruano de Audición yLenguaje.
Krashen, S. D. \&: T. D. Terrel (1983). Aprendizaje natural. Oxford: Pergamon.

Sánchez Pérez, Alquilino (2000). Los métodos en la enseñanza de idiomas. Madrid: Sociedad General Españ olade Librería S. A.

Sánchez Pérez, Alquilino (1996). El tratamiento de la gramática en una metodología integral y comun ica tiva. Murcia: Universidad de Murcia.

Sánchez Pérez, Alquilino (1992). Historia de la enseñanza del español como lengua extranjera. Madrid: SGEL

Sweet , H. (1899). Thc practica/ study of language. Florida: University Press.

Stern, H. H. (1983). Fundamental concepts of language teaching. Oxford: University Press.

Villanueva Roa, J. D. (2001). El aprendizaje de los adultos. En: htt p://www .isfti c.mepsyd.es/formacion/materialesl25 /c d/docume ntación:complementaria /1_aprendizaje_adulto s.pdf. Recuperado ell 2-ll-Ol.

Wilkins, D. (1974). Enseñanza aprendizaje de una segunda lengua. London: Arnold

Mg. Araceli Cámero Room

Magíster en Educación UNIFÉ. Licenciada enTraducción por la Universidad Ricardo Palma. 